\title{
A Deformed Shape Detection Scheme Using Time Series Images
}

\author{
Hoejung Jung* and Namgi Kim* ${ }^{1}$ \\ * Department of Computer Science, Kyonggi University, South Korea \\ junghj,ngkim@kyonggi.ac.kr
}

\begin{abstract}
Because of environmental issues such as global warming, natural disaster is a growing trend. Accordingly, interest in minimizing structural damage due to natural disasters is also increasing. One method for reducing these problems of disaster damage is to detect problematic situations quickly using Closed-circuit televisions (CCTVs), which are image sensors that allow damage to be controlled and countermeasures to be prepared. In the case of structural deformation situations occurring due to natural disasters, deformations tend to continue until the structure is recovered. However, since intelligent CCTVs have thus far failed to recognize or track moving objects, these CCTVs are not suitable for detecting the maintenance of structure deformation. Therefore, in the present study, a technique will be presented through which structural situations suspected of deformation (i.e., situations in which problems will occur if the current state is maintained) can be intelligently detected using difference images based on video images taken from actual structures at fixed positions.
\end{abstract}

Keywords: CCTV, Video Analysis, detection

\section{Introduction}

Due to environmental problems, such as global warming, the incidence of natural disasters (e.g., earthquakes, strong winds, and heavy rainfalls including typhoons) is increasing worldwide, causing increasing damage to human life and property [1]. In this situation, to minimize damage problems, CCTVs that are capable of quickly recognizing structural damage situations - and, thus, of helping to establish countermeasures against damage in problematic situations and to control damage-have begun to attract attention. The scale of the CCTV markets is expected to grow from $\$ 105$ billion dollars in 2011 to \$205 billion dollars in 2016, representing a high 14\% annual average growth rate and resulting in a large- scale market [2].

Intelligent CCTV technologies that have been used thus far include motion detection technologies, which detect objects by sensing pixel changes, and Video Analysis technologies, which detect events by tracking backgrounds and moving objects separately. These existing technologies are focused on recognizing moving objects in order to trace their movements. However, in the case of structural deformation damage situations, since such situations are maintained until the structures are recovered from damage, detecting the long-term persistence of deformation is more important than detecting the movements. Therefore, in the present paper, in Section 3, a technique applicable to CCTVs is presented for recognizing changes in video image data and for recognizing and expressing structure situations suspected of deformation in cases where changes are maintained. In addition, threshold values for changes and maintenance are applied to the presented technique so that intelligent CCTV systems can recognize structural deformation, while excluding the movements of other objects, such as cars and humans. In Section 4, deformation situations are applied to image data extracted from

\footnotetext{
${ }^{1}$ Corresponding author: Namgi Kim, E-mail: ngkim@kyonggi.ac.kr
} 
videos taken from actual structures at fixed positions to examine appropriate threshold values applicable to the presented technique. Moreover, in to conduct experiments - and, thereby, determine the results of the application of the technique-scenarios are defined for the various situations that may occur. In Section 5, conclusions and potential directions for future studies are addressed.

\section{Related Work}

Motion Detection is a technology used in the early stage of intelligent CCTVs to detect pixel changes in moving objects from CCTV videos filmed at fixed positions. This technology does not analyze target objects to detect moving objects; instead, it simply detects pixel movements on CCTV videos in order to detect moving objects. In addition, detection levels are manually set, and the sensitivity is comprehensively adjusted. Therefore, this technology may issue incorrect reports in low- illumination environments, may be vulnerable to periodic movements (such as tree movements), and cannot easily respond to weather changes [3].

Video Analysis is a technology that is more developed than Motion Detection. It, detects changes on the screen, separates the background and the moving objects, and track objects to display them on the screen. Since this technology separates background areas, it is better than motion detection with regard to detecting signal changes. It also involves relatively fewer wrong alarms and non-detection instances. However, it tends to change the shapes of moving objects rapidly. Extracted movements of objects tend to be partial or generally overlapping. Parts of objects are not visible because they are overlapped or because a single object is viewed as multiple objects due to surrounding conditions. Sizes and shapes vary by object, leading to significant deformation. For instance, in the case of quickly moving objects, information on objects being tracked is often lost. Since variables like diversely changing lighting environments and shadows act as noise in CCTV videos, parts of no interest that are not objects may be mistaken as objects. This Video Analysis technology enables the detection of diverse events in videos and the analysis of CCTV video objects; thus, it is used in the fields of video security and Business Intelligence, among others [4-5].

The suspected structure deformation expression technique presented in the present study is also a type of Video Analysis. Since this technology separates backgrounds from subjects suspected of deformation by using different images applied with threshold values, this technology is stronger against background changes than simple Motion Detection. In existing Video Analysis, the recognition of moving objects and the tracking of movements are major matters of interest, since such information is important for violent crimes, intelligent traffic systems, and parking and stopping management. However, in cases in which structures at fixed positions have been deformed by, for example, natural disasters, movements of subjects are not important, since the focus is on recognizing the parts in which the deformation is maintained in order to repair the structure deformation quickly. That is, in the case of structures that are basically fixed, persistency is more important than the movement of deformation. Therefore, the recognition of structure deformation requires the tracking of subjects of interest, which cannot be done using existing Video Analysis methods.

\section{A Deformed Shape Detection Scheme}

Within structure deformation, situations often occur outdoors due to such reasons as natural disasters inducing rapid changes. The resultant damaged parts are maintained until the structure is recovered. Therefore, in the present study, as a method of 
recognizing rapid changes in situations, the RGB color-difference formula, shown in equation (1), was applied to an RGB model [6].

$$
\Delta E_{R G B}=\sqrt{\Delta R^{2}+\Delta G^{2}+\Delta B^{2}}
$$

The RGB model is a basic color model that considers the colors of pixels to be combinations of three components: $\mathrm{R}(\mathrm{Red}), \mathrm{G}(\mathrm{Green})$, and $\mathrm{B}$ (Blue). That is, in RGB models, each pixel has R, G, and B color information. In this case, each of the R, G, and $B$ values can exist in a range between 0 and 255. Each RGB color-difference formula compares the R, G, and B values of two different pixels. When there are two pixels $p_{1}$ and $\mathrm{p}_{2}$, with different sets of information, let us assume that the RGB value of $\mathrm{p}_{1}$ is $\left(R_{1}, G_{1}, B_{1}\right)$ and that the RGB value of $\mathrm{p}_{2}$ is $\left(R_{2}, G_{2}, B_{2}\right)$. In this case, the $\Delta E_{R G B}$ of the two pixels is $\sqrt{\left(R_{2}-R_{1}\right)^{2}+\left(G_{2}-G_{1}\right)^{2}+\left(B_{2}-B_{1}\right)^{2}}$. That is, RGB color -difference formulas convert the $3 \mathrm{D}$ vector color information values into scalar values so that differences between the two pixels can be quantitatively expressed.

The suspected structure deformation expression technique applied through an RGB color-difference formula consists of four stages, as shown in Figure 1, and utilizes pseudo codes, as shown in Figure 2. The first stage is extracting the image frames $\mathrm{F}=\left\{f_{1}, f_{2}, f_{3}, \ldots, f_{i}\right\}$ from the CCTV at intervals of time $\Delta t$. In $\mathrm{F}$, if the image frame at the current time is assumed to be $f_{\mathrm{t}}$, image frames $f_{\mathrm{t}-2}, f_{\mathrm{t}-1}$ and $f_{\mathrm{t}}$, including the current image frame, will be extracted first at intervals of $\Delta \mathrm{t}$.

The second stage is to recognize suspected structural deformations, which occur when the pixel difference between $f_{\mathrm{t}-2}$ and $f_{\mathrm{t}-1}$ that is extracted first among the image frames $f_{\mathrm{t}-2}, f_{\mathrm{t}-1}, f_{\mathrm{t}}$ exceeds the threshold value. For example, if the pixel of the coordinate $(\mathrm{x}$, y) of image frame $f_{i}$ is assumed to be $P_{i}(\mathrm{x}, \mathrm{y}), P_{i}(\mathrm{x}, \mathrm{y})$ will have $3 \mathrm{D}$ vector RGB color information $\left(\mathrm{R}_{i}, G_{i}, B_{i}\right)$. Therefore, if a certain coordinate $(\mathrm{x}, \mathrm{y})$ pixel of $f_{\mathrm{t}-2}, f_{\mathrm{t}-1}$ is applied to the RGB color-difference formula, the quantitative difference value $\Delta E_{R G B}$ can be obtained. $P_{\mathrm{t}-1}(\mathrm{x}, \mathrm{y})$ is regarded as a suspected pixel only when the obtained $\triangle E_{R G B}$ is larger than the threshold value set. This is done to avoid addressing minor changes.

The third stage is to exclude those parts among the parts that are suspected to be structural deformations that are not maintained in $f_{\mathrm{t}}$. In the case of objects that move significantly and continuously, such as cars, a pixel that became a suspected pixel in the second stage (due to movement) may be recognized as a motion pixel, since $P_{t-1}(\mathrm{x}, \mathrm{y})$ is not maintained as $P_{t}(\mathrm{x}, \mathrm{y})$ of $f_{\mathrm{t}}$ and $P_{t}(\mathrm{x}, \mathrm{y})$ is excluded from the suspected pixel. On the other hand, when $P_{t-1}(\mathrm{x}, \mathrm{y})$ has been maintained as $P_{t}(\mathrm{x}, \mathrm{y})$ of $f_{\mathrm{t}}, f_{\mathrm{t}} P_{t}(\mathrm{x}, \mathrm{y})$ is confirmed as a suspected pixel. In this process, to reduce the sensitivity to changes in illumination, pixel values are regarded to have been maintained, not when they have been maintained completely equally, but when the $\Delta E_{R G B}$ obtained by substituting the two pixels into the RGB color-difference formula is smaller than 1.

In the last stage, only those suspected pixels that are confirmed in the third stage are expressed on $f_{\mathrm{t}}$ in black, representing those parts that are suspected to be structural deformations 


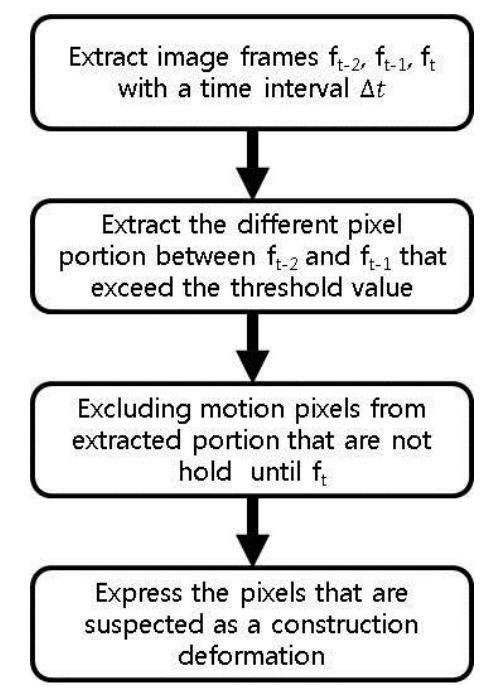

Figure 1. Proposed Process Flowchart

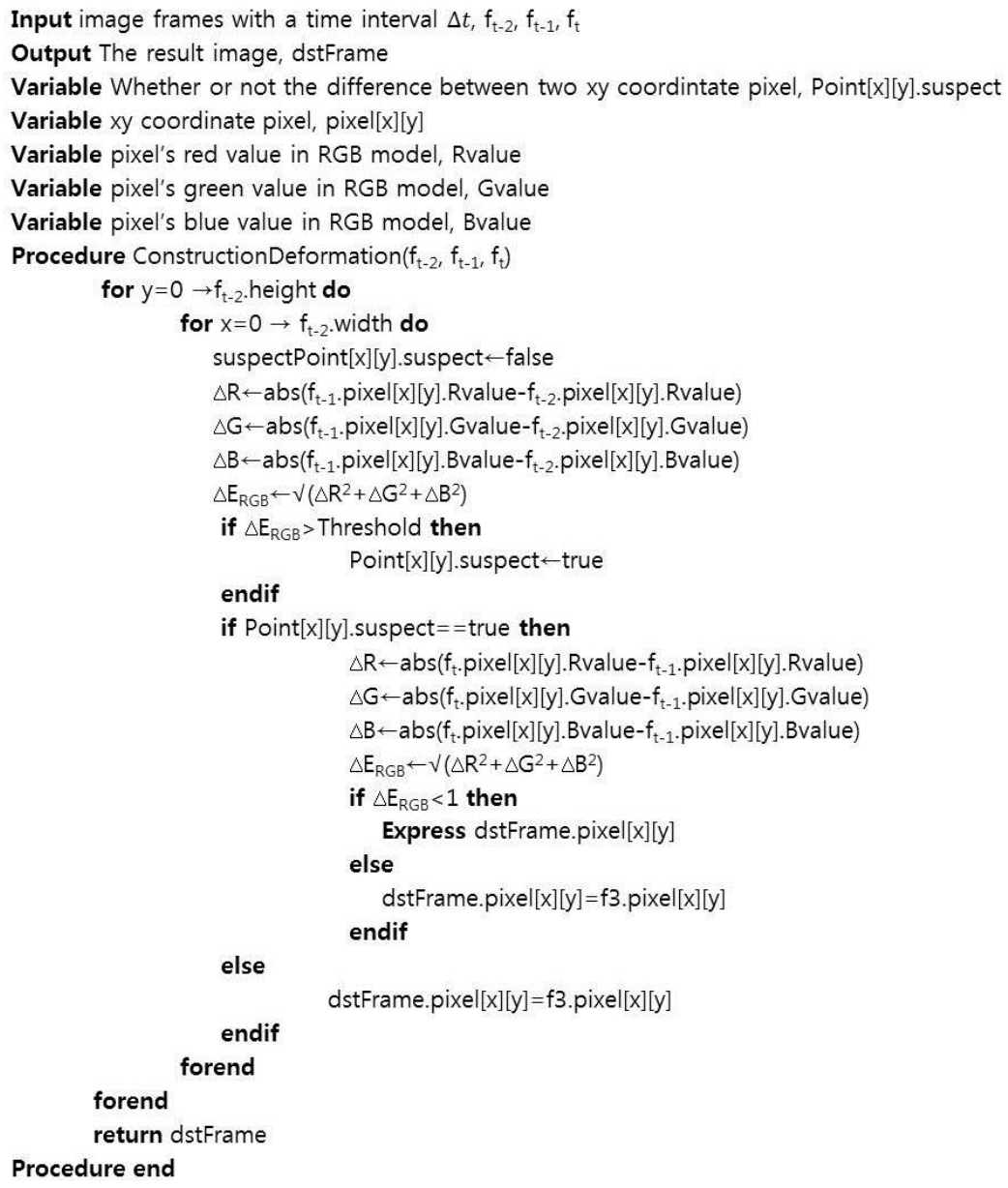

Figure 2. Proposed Process Pseudo Code 


\section{Experiment}

To examine the results of the technique to express structure situations suspected of deformation, experiments were conducted based on the image data shown in Figure 3, which were extracted from full $\mathrm{HD}$ videos that took images of the structure in Gwanggyeo Reservoir in Suwon at intervals of one second. At this time, since structural damage situations could not actually be made, a virtual deformation situation was formed in the image data in Region A in Figure 3.

In Section 4.1, three scenarios that could occur were defined as experimental subjects. To determine the appropriate threshold values to be used in the proposed technique, threshold values were changed in S3, which is a scenario environment in which deformation situations are expressed to obtain resultant images. Thereafter, in Section 4.2, the degrees of expression of the images were analyzed to determine appropriate threshold values. Finally, in Section 4.3, the resulting threshold values were applied to determine the results of the technique to express situations suspected of deformation by scenario

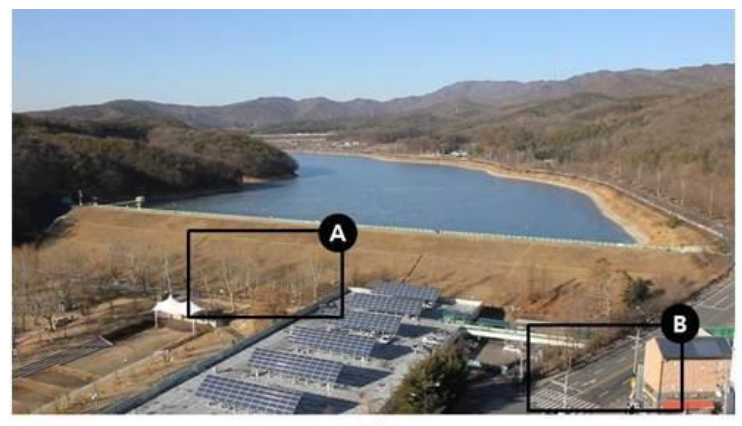

t1

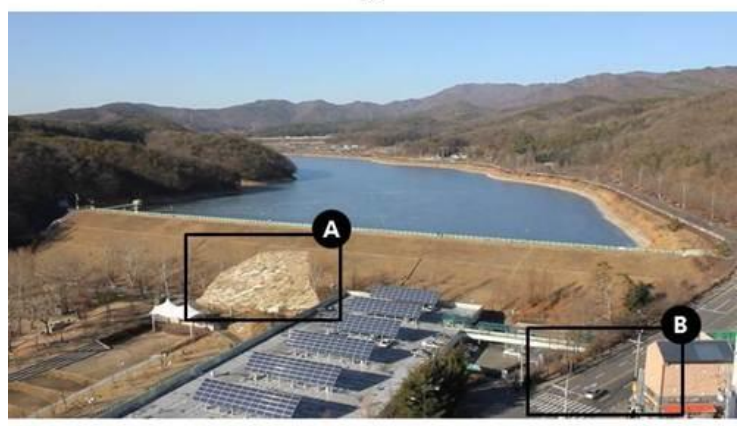

t2

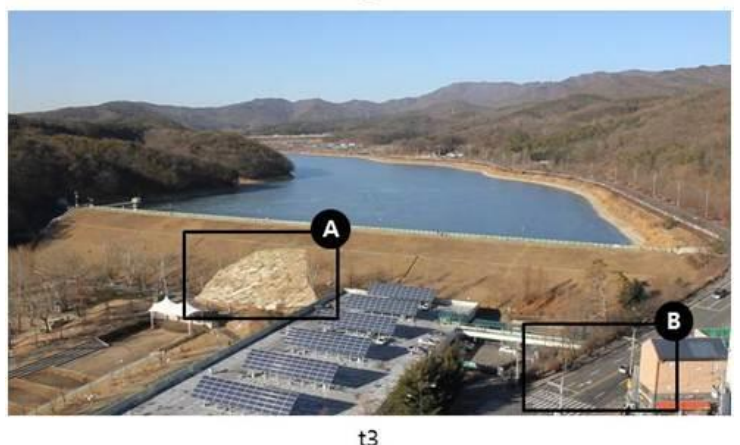

Figure 3. Experimental Image Subject Extracted from the Video at Intervals of $\Delta t=1$ 


\subsection{Definition of the Scenarios}

Before experimenting with the presented technique, scenarios S1, S2, and S3, which could occur when situations suspected of deformation were detected, were defined as follows. The pixel color graph of a certain coordinate $(x, y)$ pixel in relation to time is shown below.

$$
\text { Pixel color }=\sqrt{R^{2}+G^{2}+B^{2}}
$$

4.1.1. S1: This is a scenario for cases in which no situation suspected of deformation existed. In the test subjects of scenario S1, there is no significant difference between the pixels of the image frames during the first time $\mathrm{t} 1$ and those during time $\mathrm{t} 2$. In addition, there is no significant difference between the image frames during time $t 2$ and those during time $\mathrm{t} 3$. Therefore, the color scalar values of the coordinate $\mathrm{x}=733, \mathrm{y}=712$ pixel in the area where the deformation situation was later formed do not show any changes larger than the threshold value, as shown in Figure 4.

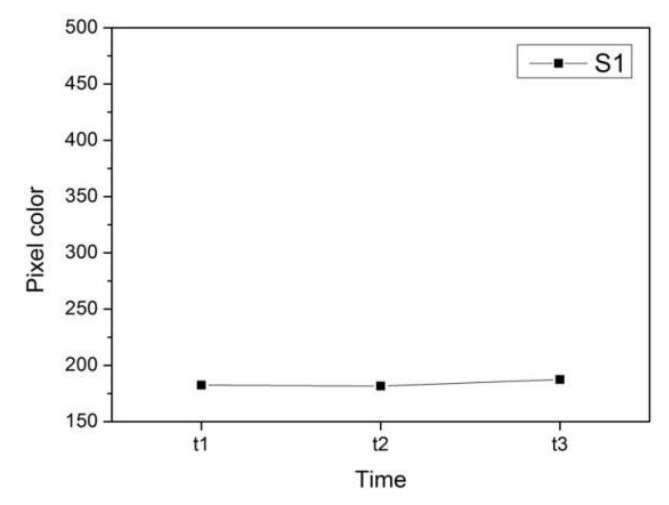

Figure 4. Changes in Coordinate $x=733, y=712$ Pixel Scalar Values in
Scenario $S 1$

4.1.2. S2: This is a scenario for cases in which situations were suspected of deformation, but the situations were not maintained. This is a scenario that targets moving subjects, such as cars and humans (as with region B in Figure 3), in which significant differences were found between the values of the pixels of the image frames during the first time $t 1$ and those during the second time $\mathrm{t} 2$. However, the differences are not maintained in the frames during time 3 . The states in which the differences are not maintained include both increases (to exceed the threshold value) and decreases in the values of the pixels of the image parts with differences, as shown in Figure 6.

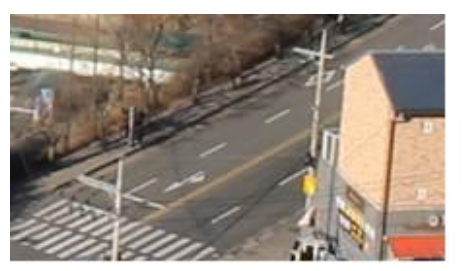

t1

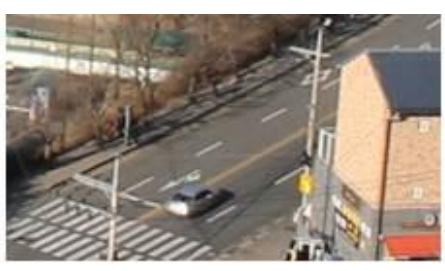

t2

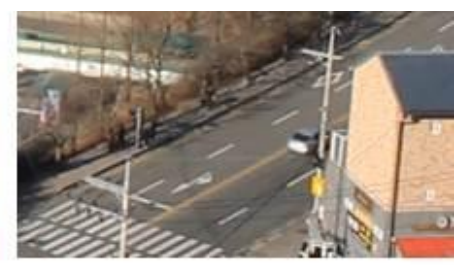

t3

Figure 5. Scenario S2's Test Subject 


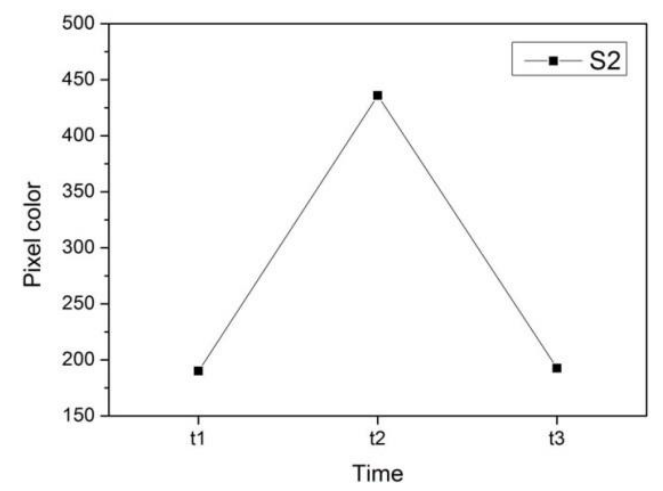

Figure 6. Changes in Coordinate $x=1017, y=1531$ Pixel Scalar Values in Scenario $\mathrm{S} 2$

4.1.3. S3: This is a scenario for cases in which situations suspected of deformation exist and are maintained. Such cases are identical to the case of region A in Figure 3. As shown in Figure 7, the pixels of the image frames during the first time t 1 and during the second time $\mathrm{t} 2$ of the test subject show significant differences. In addition, the differences are maintained in image frames during time $\mathrm{t} 3$. The states in which the differences appeared are maintained suggest that the pixel values of the situations suspected of deformation are maintained, as shown in Figure 8.

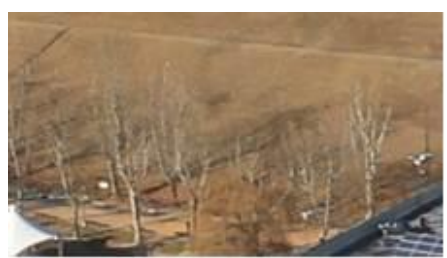

t1

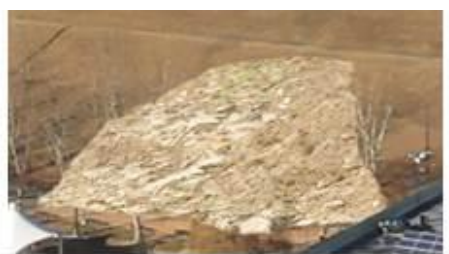

t2

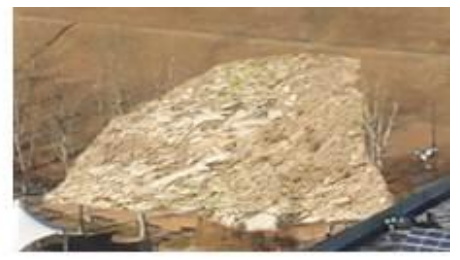

t3

Figure 7. Scenario S3's Test Subject

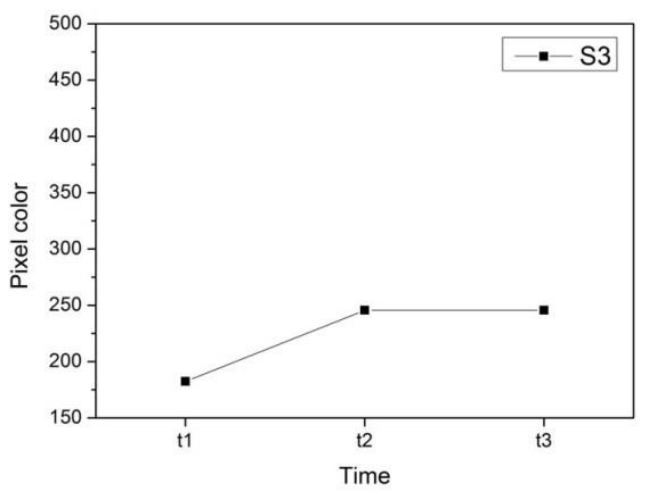

Figure 8. Changes in Coordinate $x=733, y=712$ Pixel Scalar Values in Scenario S3

\subsection{Results of the Expression of structure Situations Suspected of Deformation According to Threshold Values}

In order to find appropriate threshold values for the proposed technique applied to the defined scenarios S1, S2, S3, experiments were conducted in scenario S3, in which 
deformation situations were expressed while the threshold values were changed to 1,2 , 5, 10, 20 and 50. To quantitatively analyze the accuracy of the results for the expression of situations suspected of deformation, the numbers of the EPs (expressed pixels), IEPs (invalid expressed pixels), and VEPs (valid expressed pixels) were substituted into equations (5) and (6) to obtain the IEP and VEP rates. IEP rates are the rates of incorrect expressions of parts of structures other than the deformed parts, such that lower rates indicate smaller errors. On the other hand, VEP rates are the rates of correct expressions of the deformed parts of structures, such that lower rates indicate larger errors. The IEP rates in Figure 9 decrease as the threshold value increases to $0 \%$ and the threshold value reaches 10. Moreover, the VEP rates in Figure 9 also decrease as the threshold value increases. Therefore, when only the IEP rates are considered, the threshold values of 10 , 20, and 50 (at which the IEP rates are close to 0 ) represent small errors. However, when VEP rates are also considered, it can be seen that threshold value 10 is a relatively appropriate value because its errors increase along with its threshold values. In addition, as shown in the expression results in Figure 10, it can be seen that when the threshold value decreases below 10, pixels expressed outside the deformed parts increase, and when the threshold value increases above 10, pixels expressed inside the deformed parts decrease.

$$
\begin{array}{r}
\text { IEP rate }=\frac{\text { The number of } I E P}{\text { The number of } E P} \times 100 \% \\
\text { VEP rate }=\frac{\text { The number of } V E P}{\text { The number of } E P} \times 100 \%
\end{array}
$$
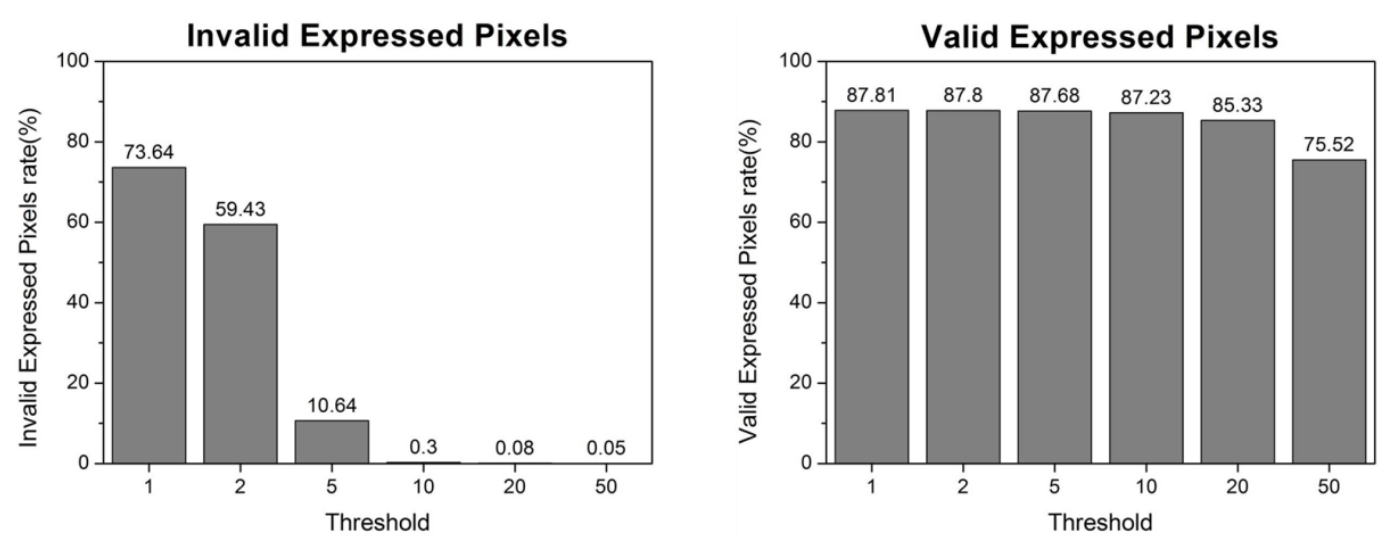

Figure 9. Graphs about Invalid Expressed Pixels and Valid Expressed Pixels 


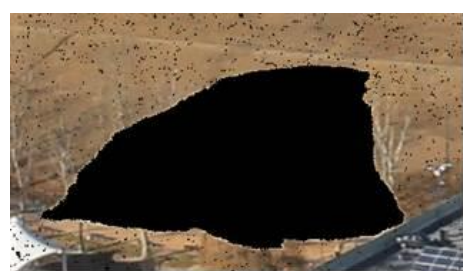

Threshold $=1$

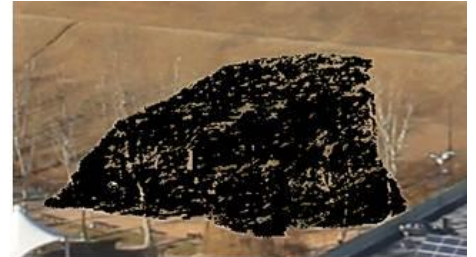

Threshold $=50$

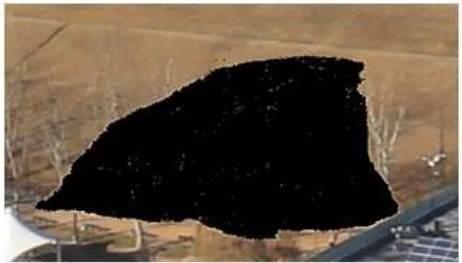

Threshold $=10$

Figure 10. Results of the Expression of Situations Suspected of Deformation in Scenario S3 when the Threshold Value was 1, 10, and 50

\subsection{Results of Expression of Structure Situations Suspected of Deformation by Scenario}

After applying the threshold value of 10 (found as set forth in Section 4.1) to the technique to express structure situations suspected of deformation, no situation suspected of deformation was detected in scenarios S1 and S2; however, a situation suspected of deformation was detected in scenario S3. The suspected part was expressed on the produced image, as shown in Figure 11.

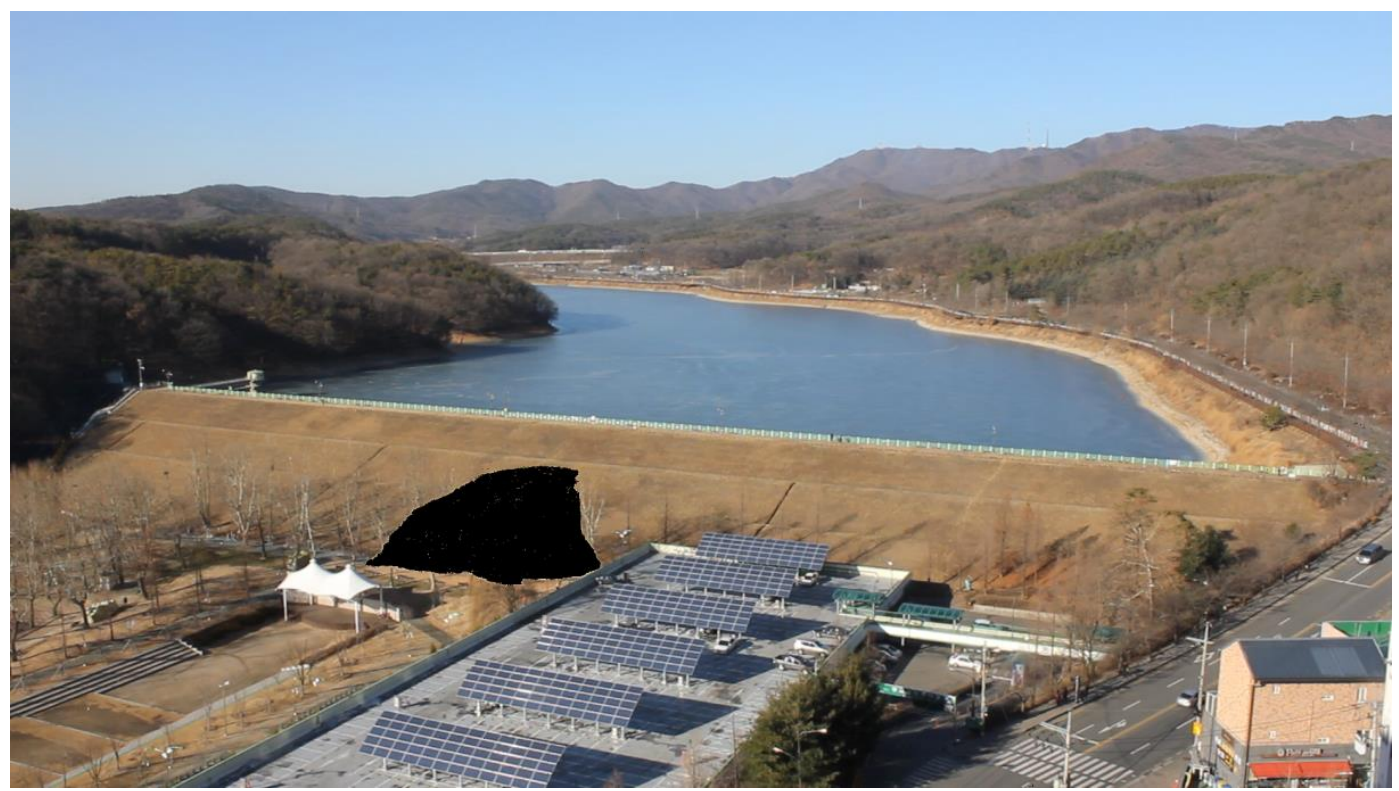

Figure 11. Result of the Expression of a Situation Suspected of Deformation in Scenario S3

\section{Conclusion}

In the present paper, a technique to detect structure situations suspected of deformation and to express the parts suspected of deformation was presented. To evaluate the performance of the presented technique, experiments were conducted using image data for actual structures. The range of the experiments was designated by 
defining scenarios for cases that could occur. Before the scenarios were tested, experiments were conducted to obtain appropriate threshold values to be used in the technique to express situations suspected of deformation. In addition, the results of experiments for various threshold values were quantitatively analyzed to determine appropriate threshold values - and, based on the threshold values, the results of the expression of situations suspected of deformation were experimented, and the experimental results were analyzed by scenario.

In future studies, the technique presented in the present paper will be applied to CCTV systems by monitoring actual waterside structures at fixed positions. Although $\Delta \mathrm{t}$ was assumed to be one second in the present study, in the case of CCTV systems that will be applied with the algorithm, variability will be given to $\Delta$ t so that the CCTV systems can be operate satisfactorily in cases of illumination and obstacles of no interest.

\section{Acknowledgements}

This research was supported by a grant (13SCIPA01) from Smart Civil Infrastructure Research Program funded by Ministry of Land, Infrastructure and Transport(MOLIT) of Korea government and Korea Agency for Infrastructure Technology Advancement(KAIA).

\section{References}

[1] P. A. Odjugo, "GLOBAL NATURAL DISASTERS AND THEIR IMPLICATIONS ON HUMAN SUSTAINABILITY”, Journal of Sustainable Development and Environmental Protection (2012).

[2] IMS Research, The World Market for CCTV and Video Surveillance Equipment (2013).

[3] G. G. S. Menezes and A. G. Silva-Filho, "MOTION DETECTION OF VEHICLES BASED ON FPGA", Programmable Logic Conference (SPL), (2010), pp. 151-154.

[4] Marco Leo, P. Spagnolo, T. D'Orazio, P. L. Mazzeo and A. Distante, "Real-time smart surveillance using motion analysis", Expert systems, vol. 27, (2010), pp. 314-337.

[5] T. K. Kim, J. H. Im and J. K. Paik, "Video object segmentation and its salient motion detection using adaptive background generation", IET Electronics Letters, vol. 45, (2009), pp. 542-543.

[6] W.S. Mokrzycki and M. Tatol, "Colour difference $\Delta$ E-A survey", Machine Graphic \& Vision (2012).

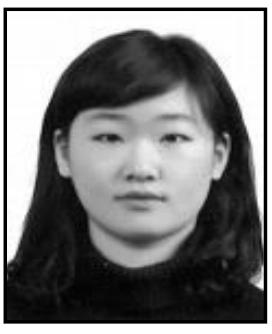

\section{Authors}

Hoejung Jung, she received the B.S. degree in Computer Science from the Kyonggi University, Korea, in 2015. She is currently M.S. candidate in Computer Science from Kyonggi University. Her research interests include computer vision, sensor networks, and wireless systems.

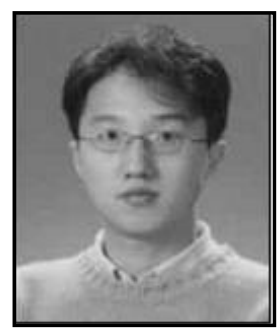

Namgi Kim, he received the B.S. degree in Computer Science from Sogang University, Korea, in 1997, and the M.S. degree and the Ph.D. degree in Computer Science from KAIST in 2000 and 2005, respectively. From 2005 to 2007, he was a research member of the Samsung Electronics. Since 2007, he has been a faculty of the Kyonggi University. His research interests include sensor system, wireless system, and mobile communication.. 